\title{
INDEX 1 FIXED POINTS OF ORIENTATION REVERSING PLANAR HOMEOMORPHISMS
}

\author{
Francisco R. Ruiz del Portal - José M. Salazar
}

\begin{abstract}
Let $U \subset \mathbb{R}^{2}$ be an open subset, $f: U \rightarrow f(U) \subset \mathbb{R}^{2}$ be an orientation reversing homeomorphism and let $0 \in U$ be an isolated, as a periodic orbit, fixed point. The main theorem of this paper says that if the fixed point indices $i_{\mathbb{R}^{2}}(f, 0)=i_{\mathbb{R}^{2}}\left(f^{2}, 0\right)=1$ then there exists an orientation preserving dissipative homeomorphism $\varphi: \mathbb{R}^{2} \rightarrow \mathbb{R}^{2}$ such that $f^{2}=\varphi$ in a small neighbourhood of 0 and $\{0\}$ is a global attractor for $\varphi$. As a corollary we have that for orientation reversing planar homeomorphisms a fixed point, which is an isolated fixed point for $f^{2}$, is asymptotically stable if and only if it is stable. We also present an application to periodic differential equations with symmetries where orientation reversing homeomorphisms appear naturally.
\end{abstract}

\section{Introduction}

Let $U \subset \mathbb{R}^{2}$ be an open set with $0 \in U$. Let $f: U \subset \mathbb{R}^{2} \rightarrow f(U) \subset \mathbb{R}^{2}$ be a homeomorphism. Let $0 \in U$ be a fixed point of $f$ which is isolated as a periodic point, i.e. there exists a neighbourhood $V \subset U$ such that $p \in V$ and $\operatorname{Fix}(f) \cap V=\operatorname{Per}(f) \cap V=\{0\}$. The fixed point index of the iterates of $f$ at $0, i_{\mathbb{R}^{2}}\left(f^{m}, 0\right)$, is a well defined integer sequence which provides important information about the local dynamical behavior of $f$ around 0 . There are results in both directions: bounds (or explicit computation) for the fixed point index

2010 Mathematics Subject Classification. 37B30, 37C25.

Key words and phrases. Fixed point index, Conley index, orientation reversing homeomorphisms, attractors, stability.

The authors have been supported by MICINN, MTM 2012-30719. 
from dynamical properties of $f$ (see for example [20], [10], [18], [13], [29], [33]) and, conversely, how the knowledge of the fixed point index is used to describe the dynamics locally (see for instance [21], [28], [31], [32]). In the abundant literature about this topic one observes that the authors restrict themselves to the orientation preserving case and the most interesting dynamical consequences are obtained when $i_{\mathbb{R}^{2}}\left(f^{m}, 0\right) \neq 1$ for some $m \in \mathbb{Z}$. Probably the most relevant result for index 1 fixed points is due to Ortega, in [25], where it is shown that for orientation preserving and area contracting diffeomorphisms, $i_{\mathbb{R}^{2}}(f, 0)=i_{\mathbb{R}^{2}}\left(f^{2}, 0\right)=1$, stability and asymptotic stability of $\{0\}$ are equivalent conditions. This theorem is not true for homeomorphisms ([1]).

In this paper we investigate the dynamical behavior near fixed points of index 1 in the orientation reversing case. The main theorem of this article is the following:

Theorem 1.1. Let $f: U \subset \mathbb{R}^{2} \rightarrow f(U) \subset \mathbb{R}^{2}$ be an orientation reversing homeomorphism, with $\operatorname{Fix}(f)=\operatorname{Fix}\left(f^{2}\right)=\{0\}$. Then, if $i_{\mathbb{R}^{2}}(f, 0)=i_{\mathbb{R}^{2}}\left(f^{2}, 0\right)=1$, there exists an orientation preserving dissipative homeomorphism $\varphi: \mathbb{R}^{2} \rightarrow \mathbb{R}^{2}$ such that for a small enough neighbourhood of $0, V_{0} \subset U,\left.\varphi\right|_{V_{0}}=\left.f^{2}\right|_{V_{0}}$ and $\{0\}$ is a global attractor for $\varphi$.

As a corollary we have that for orientation reversing planar homeomorphisms a fixed point, which is an isolated fixed point for $f^{2}$, is asymptotically stable whenever it is stable.

The paper is organized as follows: in the next section we present the preliminary definitions and techniques of prime ends and Conley index theories needed for the computations of the fixed point index. Section 3 is dedicated to the proof of Theorem 1.1 and in the last section we offer an application of our results to periodic differential equations with symmetries where orientation reversing homeomorphisms appear naturally.

The reader is referred to the text of [5], [11], [14] and [24] for information about the fixed point index theory.

\section{Preliminary definitions and local results}

Given $A \subset B \subset N, \operatorname{cl}(A), \operatorname{cl}_{B}(A), \operatorname{int}(A), \operatorname{int}_{B}(A), \partial(A)$ and $\partial_{B}(A)$ will denote the closure of $A$, the closure of $A$ in $B$, the interior of $A$, the interior of $A$ in $B$, the boundary of $A$ and the boundary of $A$ in $B$, respectively.

Let $U \subset \mathbb{R}^{2}$ be an open set. By a (local) semi-dynamical system or a local homeomorphism we mean a continuous one-to-one map $f: U \rightarrow \mathbb{R}^{2}$. A function $\sigma: \mathbb{Z} \rightarrow \mathbb{R}^{2}$ is said to be a solution to $f$ through $x$ in $N \subset U$ if $f(\sigma(i))=\sigma(i+1)$ for all $i \in \mathbb{Z}, \sigma(0)=x$ and $\sigma(i) \in N$ for all $i \in \mathbb{Z}$. The invariant part of $N$, $\operatorname{Inv}(N, f)$, is defined as the set of all $x \in N$ that admit a solution to $f$ through $x$ in $N \operatorname{Inv}^{+}(N, f)\left(\right.$ resp. $\left.\operatorname{Inv}^{-}(N, f)\right)$ will denote the set of all $x \in N$ such that 
$f^{j}(x) \in N$ for every $j \in \mathbb{N}$ (resp. $f^{-j}(x)$ is well defined and belongs to $N$ for every $j \in \mathbb{N}$ ).

A set $K \subset U$ is invariant if $f(K)=K$ and positively invariant if $f(K) \subset K$. An invariant compact set $K$ is isolated with respect to $f$ if there exists a compact neighbourhood $N$ of $K$ such that $\operatorname{Inv}(N, f)=K$. The neighbourhood $N$ is called an isolating neighbourhood of $K$.

Special cases of isolating neighbourhoods are isolating blocks. A compact set $N \subset U$ is an isolating block if $f(N) \cap N \cap f^{-1}(N) \subset \operatorname{int}(N)$.

Finally, recall that given a homeomorphism $f: U \rightarrow f(U)$ and $x \in U$, the omega limit of $x, \omega_{f}(x)$, is the set of all limit points of the subsequences of $\left\{f^{m}(x)\right\}_{m \in \mathbb{N}}$.

A fixed point $p$ is a local attractor if there exists a neighbourhood $V \subset U$ of $p$ such that for every $x \in V$, the omega limit $\omega_{f}(x)=\{p\}$. The region of attraction of $\{p\}$ is the set $A(p)=\left\{x \in U: \omega_{f}(x)=\{p\}\right\}$. When $U=\mathbb{R}^{2}$ and $f: \mathbb{R}^{2} \rightarrow \mathbb{R}^{2}$ is a homeomorphism, we say that $p$ is a global attractor if $A(p)=\mathbb{R}^{2}$.

The fixed point $p$ is said to be Lyapunov stable or simply stable, if there exists a basis of neighbourhoods of $p$ formed by positively invariant sets. The fixed point $p$ is asymptotically stable if it is a stable attractor. Analogously a fixed point is a repeller if it is asymptotically stable for $f^{-1}$.

A homeomorphism $f: \mathbb{R}^{2} \rightarrow \mathbb{R}^{2}$ is said to be dissipative if the infinity point is a repeller. Equivalently, there exists a positively invariant compact set $M \subset \mathbb{R}^{2}$ such that for every $x \in \mathbb{R}^{2}$ there exists $m=m(x) \in \mathbb{N}$ with $f^{m}(x) \in M$.

2.1. Prime ends theory. A Jordan curve $\gamma$ is a non-self-intersecting continuous loop in the plane. Every Jordan curve decompose the plain into two components. A Jordan domain is the bounded component of the complement of a Jordan curve.

Let $B \subset \mathbb{C}$ be the unit open disc and let $f: B \rightarrow G \subset \mathbb{C} \cup\{\infty\}$ be a conformal mapping. The problem whether $f$ admits an extension to $\operatorname{cl}(B)=B \cup S^{1}$, by defining $f(z)=\lim _{x \rightarrow z} f(x)$ for $z \in S^{1}$, has a topological answer. Indeed, $f$ admits and extension if and only if $\partial(G)$ is locally connected. The problem whether $f$ has an injective extension has also an answer of topological nature: $f$ has an injective extension if and only if $\partial(G)$ is a Jordan curve (Carathéodory's Theorem, see [27]). If $\partial(G)$ is locally connected but not a Jordan curve there are points of $\partial(G)$ that have several pre-images. The situation becomes much more complicated if $\partial(G)$ is not locally connected. Carathéodory introduced the notion of prime end to describe this setting. The points $z \in S^{1}$ correspond one-to-one to the prime ends of $G$ and the limit $f(z)$ exists if and only if the prime end has only one point (Prime End Theorem, see [27]).

In order to make this paper as self-contained as possible we will recall here the main definitions of the theory of ends that we will need. The reader is referred 
to the text of [22] and [27] for detailed expositions with different approaches of this theory.

Let $G \subset \mathbb{R}^{2}$ be a simply connected open domain containing the point at infinity such that $\partial(G)$ contains more than one point. Then $\partial(G)$ is bounded. A cross-cut is a simple arc, $C$, lying in $G$, except in the end points, with different extremities. If $C$ is a cross-cut of $G$ then $G \backslash C$ has exactly two components $A_{1}$ and $A_{2}$ such that $G \cap \partial\left(A_{1}\right)=G \cap \partial\left(A_{2}\right)=C \backslash\{$ end points $\}$.

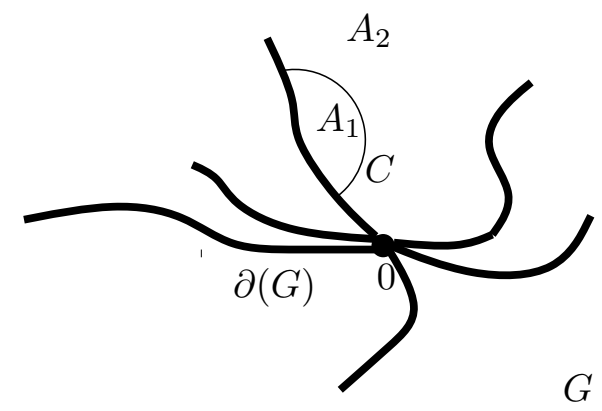

FiguRE 1

A sequence $\left\{C_{n}\right\}$ of mutually disjoint cross-cuts and such that each $C_{n}$ separates $C_{n-1}$ and $C_{n+1}$ is called a chain. A chain of cross-cuts induces a nested chain of domains (bounded by each $\left.C_{n}\right) \ldots D_{n+1} \subset D_{n} \ldots$ Each chain of crosscut defines an end. Two chains of cross-cuts, $\left\{C_{n}\right\}$ and $\left\{C_{n}^{\prime}\right\}$, are equivalent if for any $n \in \mathbb{N}$ there is $m(n)$ such that $D_{m} \subset D_{n}^{\prime}$ and $D_{m}^{\prime} \subset D_{n}$ for every $m>m(n)$. Equivalent chains of cross-cuts are said to induce the same end. If $P$ and $Q$ are ends represented by chains of cross-cuts $\left\{C(P)_{n}\right\}$ and $\left\{C(Q)_{n}\right\}$ such that for every $n, D(P)_{m} \subset D(Q)_{n}$ for $m>m(n)$ we say that $P$ divides $Q$. A prime end $P$ is an end which can not be divided by any other.

Let $P$ be a prime end. The set of points of $P$ is the intersection $E=$ $\bigcap_{n \in \mathbb{N}} \operatorname{cl}\left(D(P)_{n}\right)$ where $\left\{D(P)_{n}\right\}$ is the sequence of domains bounded by any sequence of cross-cuts representing $P$. A principal point of $P$ is a limit point of a chain of cross-cuts representing $P$ tending to a point. The set $H_{P} \subset E$ of principal points of a prime end $P$ is a continuum (compact connected set) (see [6] or [7] for details).

Each chain of cross-cuts inducing a prime end $P$ determines a basis of neighbourhoods of $P$. We obtain in this way a topology in the set of prime ends of $G$. More precisely, if $\mathbb{P}$ is the set of prime ends of $G$ and $G^{*}$ is the disjoint union of $G$ and $\mathbb{P}$, we can introduce a topology in $G^{*}$ in such a way that it becomes homeomorphic to the closed disc and the boundary being composed by the prime ends. It is enough to define a basis of neighbourhoods of a prime end $P \in \mathbb{P}$. Given the sequence of domains $\left\{D(P)_{n}\right\}$, we produce a basis of neighbourhoods 
$\left\{U_{n}\right\}$ of $P$ in $G^{*}$. Each $U_{n}$ is composed by the points in $D(P)_{n}$ and by the prime ends $Q$ such that $D(Q)_{m} \subset D(P)_{n}$ for $m$ large enough.

A point $y \in \partial(G)$ is accessible if there exists an arc $\gamma:[0,1] \subset \operatorname{cl}(G)$ such that $\gamma(1)=y$ and $\gamma([0,1)) \subset G$. Each accessible point determines a prime end.

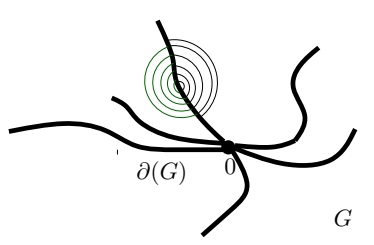

(a)

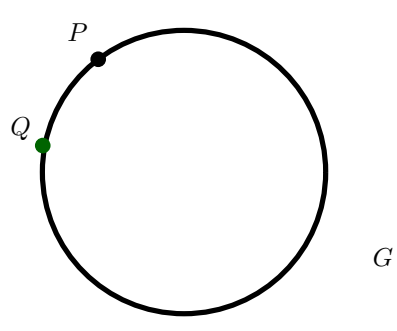

(b)

Figure 2. (a) Chains of cross-cuts defining prime ends $P$ and $Q$. (b) Prime ends $P$ and $Q$ in the set of prime ends, $\mathbb{P}$, of $G$.

If $S^{2}$ is the 2-sphere $\mathbb{R}^{2} \cup\{\infty\}$ and $\infty \in D \subset S^{2}$ is a simply connected open domain, the natural compactification, due to Carathéodory, see [6], of $D$ obtained by attaching to $G$ a set homeomorphic to the one-dimensional sphere $S^{1}$ is called the prime ends compactification of $G$. We identify $\mathbb{R}^{2}=\mathbb{C}$ and we consider a conformal homeomorphism $g: G \rightarrow S^{2} \backslash B$ (where $B$ is the disc $B=\{z \in \mathbb{C}:|z| \leq 1\}$ ). Now a one-dimensional sphere $S^{1}$ is attached to $G$ using $g$. Each point of $S^{1}$ corresponds to a prime end of $G$.

2.2. Conley index tools. The next definition is based in the notion of filtration introduced by J. Franks and D. Richeson, in [12], and it is the key for the direct computation of the fixed point index of any iteration of any homeomorphism of the plane.

Definition 2.1. Let $f: U \subset \mathbb{R}^{2} \rightarrow \mathbb{R}^{2}$ be a local homeomorphism. Suppose that $L \subset N$ is a compact pair contained in the interior of $U$. The pair $(N, L)$ is said to be a strong filtration pair for $f$ provided $N$ and $L$ are each the closure of their interiors and

(a) $N$ and $\partial(N \backslash L)$ are homeomorphic to a disc and $S^{1}$, respectively.

(b) $\operatorname{cl}(N \backslash L)$ is an isolating neighbourhood.

(c) $f(\operatorname{cl}(N \backslash L)) \subset \operatorname{int}(N)$ (i.e. $L$ is a neighbourhood of $N^{-}$in $N$ ).

(d) For any component $L_{i}$ of $L, \partial_{N}\left(L_{i}\right)$ is an arc and there exists a topological disc $B_{i}$ such that $\partial_{N}\left(L_{i}\right) \subset B_{i} \subset L_{i}, B_{i} \cap N^{-} \neq \emptyset$ and $f\left(B_{i}\right) \cap$ $\operatorname{cl}(N \backslash L)=\emptyset$.

Next theorem is one of the more important results of [30] and asserts the existence of strong filtration pairs. 
TheOREM 2.2 ([30]). Let $f: U \subset \mathbb{R}^{2} \rightarrow \mathbb{R}^{2}$ be a local homeomorphism. Let $p \in U$ be a non-repeller fixed point of $f$ such that $\{p\}$ is an isolated invariant set. Then there exists a strong filtration pair for $f,(N, L)$, with $\{p\}=\operatorname{Inv}(\operatorname{cl}(N \backslash$ $L), f)$.

The following theorem is a generalization of a result of Le Calvez and Yoccoz in [20] which is proved in [30] or [31] using Conley index techniques.

TheOREM 2.3 ([30]). Let $f: U \subset \mathbb{R}^{2} \rightarrow f(U) \subset \mathbb{R}^{2}$ be a homeomorphism. Suppose that there exists a strong filtration pair, $(N, L)$, for $f$ and let $K=$ $\operatorname{Inv}(\operatorname{cl}(N \backslash L), f)$. Then, there are an $A R$ (an absolute retract for metric spaces), $D_{0}$, containing a neighbourhood $V \subset \mathbb{R}^{2}$ of $K$, a finite subset $\left\{q_{1}, \ldots, q_{m}\right\} \subset D_{0}$ and a map $\bar{f}: D_{0} \rightarrow D_{0}$ such that $\left.\bar{f}\right|_{V}=\left.f\right|_{V}$ and for every $k \in \mathbb{N}, \operatorname{Fix}\left((\bar{f})^{k}\right) \subset$ $K \cup\left\{q_{1}, \ldots, q_{m}\right\}$. Moreover,

(a) If $f$ preserves the orientation, then

$$
i_{\mathbb{R}^{2}}\left(f^{k}, K\right)= \begin{cases}1-r q & \text { if } k \in r \mathbb{N}, \\ 1 & \text { if } k \notin r \mathbb{N},\end{cases}
$$

where $k \in \mathbb{N}, q$ is the number of periodic orbits of $\bar{f}$ in $\left\{q_{1}, \ldots, q_{m}\right\}$ and $r$ is their period.

(b) If $f$ reverses the orientation, then

$$
i_{\mathbb{R}^{2}}\left(f^{k}, K\right)= \begin{cases}1-\delta & \text { if } k \text { odd }, \\ 1-\delta-2 q & \text { if } k \text { even }\end{cases}
$$

where $\delta \in\{0,1,2\}$ and $q$ are the number of fixed points and period two orbits of $\bar{f}$ in $\left\{q_{1}, \ldots, q_{m}\right\}$, respectively.

DEFINITION 2.4. Under the setting of the above theorem, the integer $r(r=2$ if $f$ is orientation reversing) is called the period of the strong filtration pair $(N, L)$.

EXAMPLE 2.5. Let us consider the homeomorphism $f$ of Figure 3 and the homeomorphisms $g, s: \mathbb{R}^{2} \rightarrow \mathbb{R}^{2}$ which are a $\pi / 2$-rotation and a symmetry with respect to $\{x=y\}$ respectively. Let $D_{0}$ be the quotient space obtained from $N$ by identifying each $L_{i}$ to a point $q_{i}$. The orientation preserving homeomorphism $g \circ f$ has associated a strong filtration pair $(N, L)$ (see Figure 3 ). The induced map $\overline{g \circ f}: D_{0} \rightarrow D_{0}$ has $q=1$ periodic orbits of period $r=4$ in $\left\{q_{i}\right\}_{i}(r=4$ is the period of the strong filtration pair). Then,

$$
i_{\mathbb{R}^{2}}\left((g \circ f)^{k}, 0\right)= \begin{cases}1-4 & \text { if } k \in 4 \mathbb{N}, \\ 1 & \text { if } k \notin 4 \mathbb{N} .\end{cases}
$$

On the other hand, the orientation reversing homeomorphism $s \circ f$ has associated a strong filtration pair $(N, L)$ (see Figure 3 ) with $q=1$ periodic orbits of period 2 
and $\delta=2$ fixed points for the induced map $\overline{s \circ f}: D_{0} \rightarrow D_{0}$. Then

$$
i_{\mathbb{R}^{2}}\left((s \circ f)^{k}, 0\right)= \begin{cases}1-\delta=-1 & \text { if } k \text { odd }, \\ 1-\delta-2 q=-3 & \text { if } k \text { even } .\end{cases}
$$

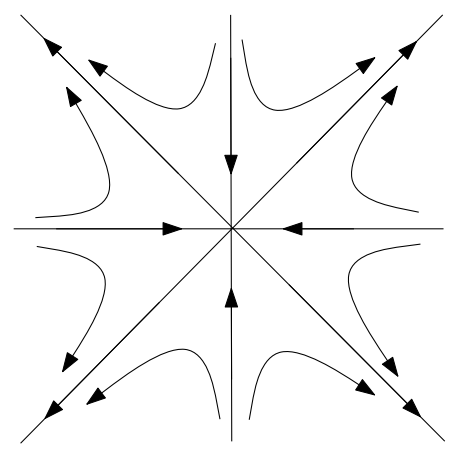

(a)

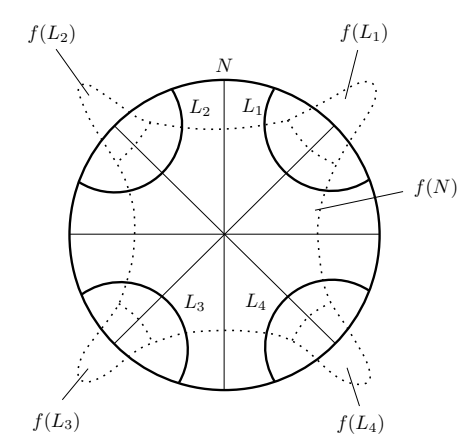

(b)

Figure 3. (a) Dynamics of $f$. (b) Strong filtration pair for $f$.

REMARK 2.6. Let $\theta=\left\{p_{1}, \ldots, p_{s}\right\}$ be the biggest subset of $\left\{q_{1}, \ldots, q_{m}\right\}$ on which $\bar{f}$ acts as a permutation. Notice that the number $s$ does not depend on the filtration but on the germ of the homeomorphism. Moreover, if $f$ is orientation preserving, $s=q r$ and the numbers $\{q, r\}$ depend on the germ of $f$. In the same way, if $f$ is orientation reversing, $s=\delta+2 q$ and the numbers $\{\delta, q\}$ depend on the germ of $f$.

This is an essential idea for computations we will use in the forthcoming results.

2.3. Computation of the index. Let $U \subset \mathbb{R}^{2}$ be an open set with $0 \in U$. Let $f: \mathbb{R}^{2} \rightarrow \mathbb{R}^{2}$ be an orientation reversing homeomorphism with $\operatorname{Fix}\left(\left.f\right|_{U}\right)=$ $\operatorname{Fix}\left(\left.f^{2}\right|_{U}\right)=\{0\}$ and such that $i_{\mathbb{R}^{2}}(f, 0)=i_{\mathbb{R}^{2}}\left(f^{2}, 0\right)=1$.

There are two situations:

Case 1. $\{0\}$ is an isolated invariant set.

Case 2. $\{0\}$ is not an isolated invariant set.

In Case 1, by Theorems 2.2 and 2.3 above, we can select a strong filtration pair $(N, L)$ with $N$ a disc and $L=\emptyset$. Then $\{0\}$ is asymptotically stable. Given an asymptotically stable fixed point $p$, the region of attraction, $A(p)$, is an open and simply connected subset of the plane. A classical result due to Kerékjártó, see [16] and [3], says that the restriction of $f$ to $A(p)$ is topologically conjugate to one of the following maps in $\mathbb{C}$,

$$
z \mapsto \frac{1}{2} z \quad \text { or } \quad z \mapsto \frac{1}{2} \bar{z} .
$$


Notice that this alternative depends on whether $f$ preserves or reverses the orientation.

The study of Case 2 needs previous work which we expose here. Suppose that $\{0\}$ is not an isolated invariant set. Since the space of components of $\operatorname{Inv}(\operatorname{cl}(J), f)$ is zero-dimensional, there exists a Jordan domain $J, \operatorname{cl}(J) \subset U$, with $0 \in J$ and such that the connected component of $K=\operatorname{Inv}(\operatorname{cl}(J), f)$ which contains the fixed point $0, K_{0}$, intersects the boundary of $J$. One has that $K_{0}$ does not decompose the plane. Moreover, by the following theorem due to K. Kuperberg [17], $0 \in \partial\left(K_{0}\right)$.

THEOREM $2.7([17])$. Let $h$ be an orientation reversing homeomorphism of the plane onto itself. If $X$ is a plane continuum invariant under $h$, then $h$ has a fixed point in $X$. Furthermore, if at least one of the bounded complementary domains of $X$ is invariant under $h$, then $h$ has at least two fixed points in $X$.

Let $\bar{f}: S^{2} \rightarrow S^{2}$ be the extended homeomorphism of $f$ to $S^{2}$. The Carathéodory's prime ends compactification of $S^{2} \backslash K_{0}$ is a disc (obtained by gluing a copy of $S^{1}$ to $S^{2} \backslash K_{0}$ ) which we call $D$. The homeomorphism $\left.\bar{f}\right|_{S^{2} \backslash K_{0}}: S^{2} \backslash K_{0} \rightarrow$ $S^{2} \backslash K_{0}$ can be extended to a homeomorphism $\widehat{f}: D \rightarrow D$. Let us denote by $\partial(D)$ the prime ends circle and consider $\mathbb{P}\left(K_{0} \cap \partial(J)\right)$ to be the set of prime ends obtained from the accessible points by $\operatorname{arcs}$ in $S^{2} \backslash \operatorname{cl}(J)$.

Since $\widehat{f}$ is orientation reversing, then $\left.\widehat{f}\right|_{\partial(D)}$ has exactly two fixed points and, eventually, 2-periodic orbits.

It is not difficult to see that the sets $\operatorname{Per}\left(\left.\widehat{f}\right|_{\partial(D)}\right)$ and $\mathbb{P}\left(K_{0} \cap \partial(J)\right)$ are disjoint.

Notice that $\widehat{f}$ and the set of periodic prime ends depend on the Jordan domain $J$ such that $K_{0} \cap \partial(J) \neq \emptyset$.

Our goal is to give a detailed description of the local dynamics of $f$ in a neighbourhood of 0 . Let us suppose first that $\operatorname{Per}\left(\widehat{f}_{\partial(D)}\right)$ is a finite set of $n=2 q+2$ points with $q$ 2-periodic orbits $\left\{\left\{q_{11}, q_{21}\right\}, \ldots,\left\{q_{1 q}, q_{2 q}\right\}\right\}$ and two fixed points $\left\{p_{1}, p_{2}\right\}$.

Let us suppose that $D \subset S^{2}$ and let us denote by $\widehat{f}_{s}: S^{2} \rightarrow S^{2}$ the homeomorphism obtained by pasting along $\partial(D)$ a symmetric copy of $\widehat{f}: D \rightarrow D$.

Lemma $2.8([32])$. Given a fixed point $p_{i}$ of $\left.\widehat{f}_{s}^{k}\right|_{\partial(D)}, k \in\{1,2\},\left(p_{i} \in\left\{p_{1}, p_{2}\right\}\right.$ if $k=1$ and $p_{i} \in\left\{\left\{q_{11}, q_{21}\right\}, \ldots,\left\{q_{1 q}, q_{2 q}\right\}\right\}$ if $\left.k=2\right)$, there is a pair $\left(N_{i}, L_{i}\right)$ which is in one of the following two situations:

(a) $\left(N_{i}, L_{i}\right)$ is a strong filtration pair for $\widehat{f}_{s}^{k}: S^{2} \rightarrow S^{2}$, in a neighbourhood of $p_{i}$.

(b) The pair $\left(N_{i}, L_{i}\right)$ has the properties (a), (b) and (c) of strong filtration pairs with $L_{i}$ an annulus, $\partial_{N_{i}}\left(L_{i}\right) \simeq S^{1}$ and $N_{i} \subset \operatorname{int}\left(\widehat{f}_{s}^{k}\left(N_{i}\right)\right)$.

Definition 2.9. We are interested, for each fixed point $p_{i}$ of $\left.\widehat{f}_{s}{ }^{k}\right|_{\partial(D)}, k \in$ $\{1,2\}$, in the pairs $\left(N_{i} \cap D, L_{i} \cap D\right)=\left(N_{i}^{\prime}, L_{i}^{\prime}\right)$ which we call strong filtration 


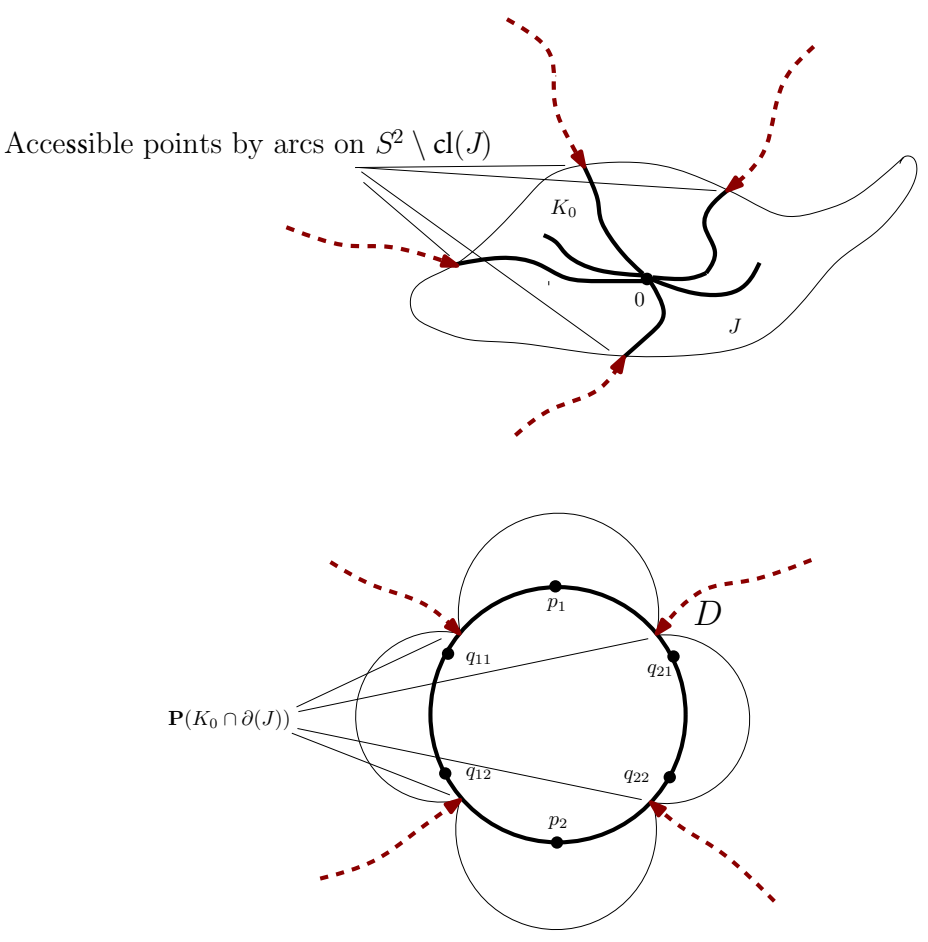

Figure 4

pairs adapted to $D$ for $p_{i}$. Notice that the pair $\left(N_{i}^{\prime}, L_{i}^{\prime}\right)$ has the properties of the strong filtration pairs for $\widehat{f}^{k}: D \rightarrow D$ at each fixed point $p_{i} \in \partial(D)$. Without loss of generality we can assume that each $\operatorname{arc} \gamma_{i}=\partial_{D}\left(N_{i}^{\prime}\right)$ corresponds in $J$ to an arc with two end points in $K_{0}$. There are three possible cases:

(a) If $L_{i}=\emptyset$, then $\widehat{f}^{k}\left(N_{i}^{\prime}\right) \subset i n t_{D}\left(N_{i}^{\prime}\right)$ and we say that $p_{i}$ is asymptotically stable for $\widehat{f}^{k}$.

(b) If $\partial_{N_{i}}\left(L_{i}\right) \simeq S^{1}$, then $N_{i}^{\prime} \subset \operatorname{int}_{D}\left(\widehat{f}^{k}\left(N_{i}^{\prime}\right)\right)$ and we say that $p_{i}$ is repeller for $\widehat{f}^{k}$.

(c) If $\left(N_{i}, L_{i}\right)$ is a strong filtration pair for $\widehat{f}_{s}^{k}$ at $p_{i}, L_{i} \neq \emptyset$, we say that $p_{i}$ is a NAR point (non-attractor/repeller) for $\hat{f}^{k}$.

REMARK 2.10. We shall make computations from indices of homeomorphisms defined in semi-discs. We will use an extension of Dancer's techniques ([9]). Given a fixed point, $p_{i}$, for $\left.\widehat{f}_{s}^{k}\right|_{\partial(D)}, k \in\{1,2\}$, and given a strong filtration pair, $\left(N_{i}, L_{i}\right)$, if $L_{i}$ has $m_{i}$ connected components $L_{i}=\bigcup_{j=1}^{m_{i}} L_{i}^{j}$, after identification to points we obtain the biggest permutation, $\theta\left(p_{i}\right)$, for the induced map $\overline{\widehat{f}_{s}^{k}}: N_{i} / \sim \rightarrow N_{i} / \sim, k \in\{1,2\}$. The set $N_{i} / \sim$ is the quotient space obtained 


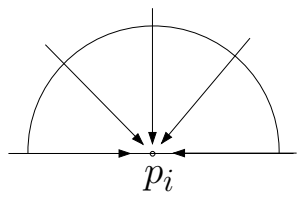

(a)

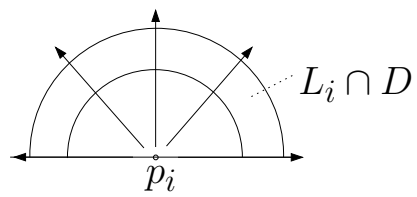

(b)

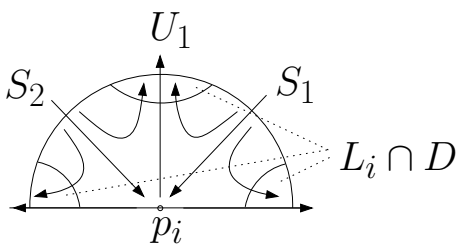

(c)

FiguRe 5

from $N_{i}$ by identifying each $L_{i}^{j}$ to a point. We select the components of $L_{i}$, $\left\{L_{i}^{j}\right\}_{j=1}^{s_{i}}$, associated to the points of the permutation $\theta\left(p_{i}\right)$. Let us observe that:

- If $p_{i}$ is periodic of period 2 for $\widehat{f}, i_{S^{2}}\left(\widehat{f}_{s}^{2}, p_{i}\right)=1-q_{i} r_{i}=1-q_{i}$, and $s_{i}=q_{i} r_{i}=q_{i}$. Each point of $\theta\left(p_{i}\right)$ is a fixed point for $\overline{\widehat{f}_{s}^{2}}$.

- If $p_{i}$ is a fixed point for $\widehat{f}, i_{S^{2}}\left(\widehat{f}_{s}, p_{i}\right)=1-\delta_{i}-2 q_{i}$, and $s_{i}=\delta_{i}+2 q_{i}$ with $\delta_{i} \in\{0,2\}$. Each periodic orbit of $\theta\left(p_{i}\right)$ is a fixed point or a periodic orbit of period 2 for $\overline{\hat{f}_{s}}\left(\delta_{i}\right.$ fixed points and $q_{i}$ orbits of period 2$)$.

We take from this family $\left\{L_{i}^{j}\right\}_{j=1}^{s_{i}}$ the components which are contained in $D \backslash \partial(D)$ and we call them exit components for $\widehat{f}^{k}$ at $p_{i}$. If a component $L_{i}^{j}$ of the family $\left\{L_{i}^{j}\right\}_{j=1}^{s_{i}}$ intersects $\partial(D)$, then we call to $L_{i}^{j} \cap D$ boundary exit component for $\widehat{f}^{k}$ at $p_{i}$ (see Figure $5(\mathrm{c})$ ).

Definition 2.11. Given a Jordan domain $J$, a set of strong filtration pairs adapted to $D$ is a finite collection of pairs $\left\{\left(N_{i} \cap D, L_{i} \cap D\right)\right\}_{i}$ associated to the family $\left\{p_{i}\right\}_{i}$ of fixed points of $\left.\widehat{f}_{s}^{k}\right|_{\partial(D)}, k \in\{1,2\}$.

Let us observe that this set depends on the choice of $J$ and, if $\operatorname{Per}\left(\left.\widehat{f}\right|_{\partial(D)}\right)$ is not finite, on the choice of a set $I \subset \partial(D)$ such that, after an adequate identification (see Lemma 2.12), transforms $\operatorname{Per}\left(\left.\widehat{f}\right|_{\partial(D)}\right)$ in a finite set.

LEMma 2.12. If $\operatorname{Per}\left(\left.\widehat{f}\right|_{\partial(D)}\right)$ is not a finite set of points, we can select a finite and disjoint union $I=I_{1} \cup \cdots \cup I_{n}$, of closed arcs of $\partial(D)$, with $\widehat{f}(I)=I$, such that:

(a) $\operatorname{Per}\left(\widehat{f}_{\partial(D)}\right) \subset I$ and $\mathbb{P}\left(K_{0} \cap \partial(J)\right) \cap I=\emptyset$.

(b) If we identify each component of I to a point we obtain a new disc which we call $D$ again. If $\widehat{f}: D \rightarrow D$ is the new induced homeomorphism we 
have that $\operatorname{Per}\left(\left.\widehat{f}\right|_{\partial(D)}\right)$ is a finite set and the construction of the strong filtration pairs adapted to $D$ is also valid.

Proof. Since $\operatorname{Per}\left(\widehat{f}_{\partial(D)}\right)$ is a compact set contained in $\partial(D) \backslash \mathbb{P}\left(K_{0} \cap \partial(J)\right)$, there exists a finite family of open intervals $V=\bigcup_{i=1}^{m} V_{i} \subset \partial(D) \backslash \mathbb{P}\left(K_{0} \cap \partial(J)\right)$ such that $\operatorname{Per}\left(\left.\widehat{f}\right|_{\partial(D)}\right) \subset V$ and $V_{i} \cap \operatorname{Per}\left(\left.\widehat{f}\right|_{\partial(D)}\right) \neq \emptyset$ for all $i=1, \ldots, m$. We select $M=\bigcup_{i=1}^{m} M_{i}$ a finite and disjoint union of closed intervals (perhaps points) with $M_{i} \subset \stackrel{i=1}{V_{i}}$ for all $i=1, \ldots, m$ in such a way that the end-points of each $M_{i}=\left[a_{i}, b_{i}\right]$ are the extreme points of $\operatorname{Per}\left(\left.\widehat{f}\right|_{\partial(D)}\right) \cap V_{i}$. Then $I=M \cap \widehat{f}^{-1}(M)$ is the set we are looking for. The only non-trivial fact we must to prove is that $\widehat{f}(I)=I$. In fact, given $d \in I$, let $\left[d_{1}, d_{2}\right] \subset \partial(D)$ be the closed interval (perhaps a point) which contains $d$ with $\left[d_{1}, d_{2}\right] \cap \operatorname{Per}\left(\widehat{f}_{\partial(D)}\right)=\left\{d_{1}, d_{2}\right\}$. Since $d \in I$, then $\left[d_{1}, d_{2}\right] \subset I$ and $\widehat{f}\left(\left[d_{1}, d_{2}\right]\right) \subset M_{j}$ for some $j=1, \ldots, m$. On the other hand, $\widehat{f}^{2}\left(\left[d_{1}, d_{2}\right]\right)=\left[d_{1}, d_{2}\right]$. Consequently, $\widehat{f}(d) \in M \cap \widehat{f}^{-1}(M)=I$, that is, $\widehat{f}(I) \subset I$. Moreover, since $\widehat{f}(d) \in I, \widehat{f}\left(\left[d_{1}, d_{2}\right]\right) \subset I$ and there exists $d_{0} \in \widehat{f}\left(\left[d_{1}, d_{2}\right]\right) \subset I$ such that $\widehat{f}\left(d_{0}\right)=d$, then $I \subset \widehat{f}(I)$.

EXAmple 2.13. Let us consider the dynamical system of Figure 6 with $K_{0}=\operatorname{Inv}(\operatorname{cl}(J), f)$. The dynamics of $\widehat{f}$ in $D$ is given in Figure $7(\mathrm{a})$. The new homeomorphism $\widehat{f}$ obtained after identification of the components of $I=I_{1} \cup I_{2}$ to points $p_{1}$ and $p_{2}$ appears in Figure 7(b) and has a repelling petal in $p_{1}$ and an unstable branch in $p_{2}$. Let us observe that the choice of the invariant intervals which contain the fixed prime ends, $I=I_{1} \cup I_{2}$ is not unique. We can select $I$ with an arbitrary family of intervals which give us a different dynamic for $\widehat{f}$ and a different set of fixed and periodic points in $\partial(D)$ for the identification map.

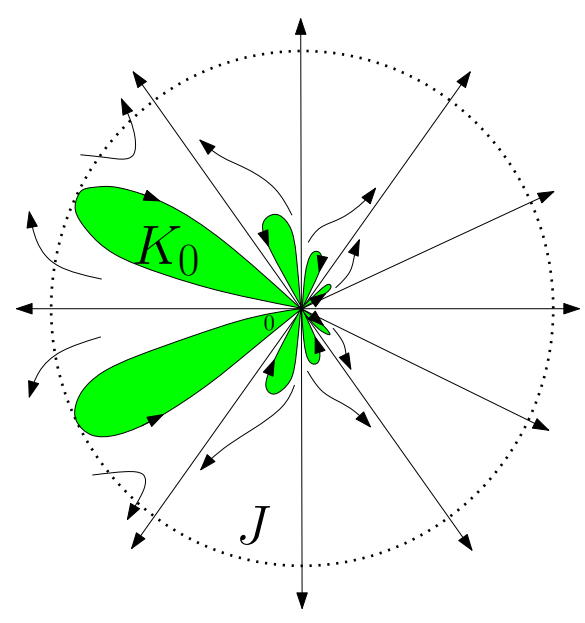

Figure 6 


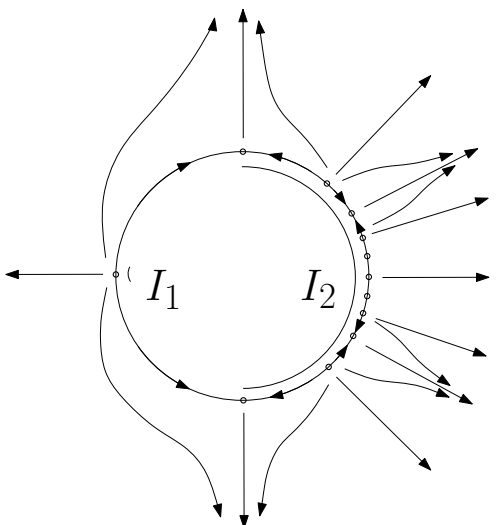

(a)

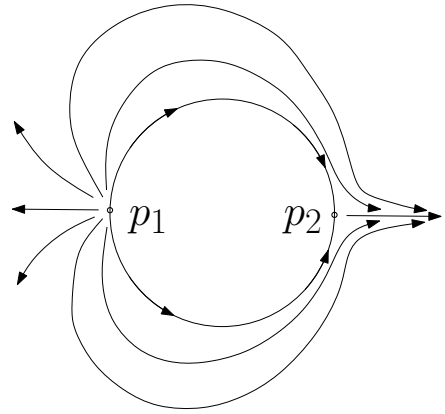

(b)

FiguRe 7

REMARK 2.14. From now onwards we will suppose that, after identification if necessary, $\operatorname{Per}\left(\left.\widehat{f}\right|_{\partial(D)}\right)$ is a finite set of two fixed points $\left\{p_{1}, p_{2}\right\}$ and $q$ periodic orbits of period $2,\left\{q_{1 j}, q_{2 j}\right\}_{j}$. Moreover, the last lemma, in order to simplify computations, permit us suppose that $\operatorname{Per}\left(\widehat{f}_{\partial(D)}\right)=\left\{p_{1}, p_{2}\right\}$.

THEOREM 2.15 (Poincaré formula. Orientation reversing case[32]). Let $f: U \subset$ $\mathbb{R}^{2} \rightarrow f(U) \subset \mathbb{R}^{2}$ be an orientation reversing local homeomorphism with 0 a fixed point which is not an isolated invariant set but isolated as periodic orbit. Let us select a Jordan domain $J$ such that $0 \in J \subset \operatorname{cl}(J) \subset U$, with $K_{0} \cap \partial(J) \neq \emptyset$ ( $K_{0}$ is the connected component of $K=\operatorname{Inv}(\operatorname{cl}(J), f)$ which contains 0$)$, and let $\left\{\left(N_{i} \cap D, L_{i} \cap D\right)\right\}_{i}$ be a set of strong filtration pairs adapted to $D$, the Carathéodory's compactification of $S^{2} \backslash K_{0}$. Then there exist $u_{0}, u_{0}^{\prime}, r_{0}, r_{0}^{\prime} \in \mathbb{N}$ with $u_{0}^{\prime} \leq u_{0}, r_{0}^{\prime} \leq r_{0}, u_{0}^{\prime}+r_{0}^{\prime} \leq 2$ such that

$$
i_{\mathbb{R}^{2}}\left(f^{k}, 0\right)= \begin{cases}1-u_{0}+r_{0} & \text { if } k \text { even } \\ 1-u_{0}^{\prime}-r_{0}^{\prime} & \text { if } k \text { odd }\end{cases}
$$

The dynamical meaning is the following: there are $u_{0}$ exit components for $\widehat{f}^{2}$ at the periodic points of $\partial(D)$ (only fixed points if we consider the identification of Remark 2.14). Moreover, $u_{0}^{\prime} \leq 2$ of the components are exit components for $\widehat{f}$ at $u_{0}^{\prime}$ fixed points for $\widehat{f}$. These $u_{0}^{\prime}$ exit components are invariant for $\widehat{\widehat{f}}$ after identification to points. In the same way there are $r_{0}$ fixed points for $\widehat{f}^{2}$ in $\partial(D)$ which are repeller for $\widehat{f}^{2}$ at $D$ and $r_{0}^{\prime} \leq 2$ of them are repeller for $\widehat{f}$ at $D$.

\section{Proof of Theorem 1.1}

We must construct the map $\varphi$. Let us suppose, without loss of generality, that $f^{2}$ is defined in the closed ball $U_{0}=B(0,1) \subset U$. We also suppose that $\{0\}$ 
is not a compact and isolated invariant set (the argument for the case of $\{0\}$ an isolated and invariant set is a consequence of the fact that $f^{2}$ is locally conjugated with $g(x)=x / 2)$.

Let $J$ be a Jordan domain such that $\operatorname{cl}(J) \subset U_{0}, 0 \in J$ and the connected component of $K=\operatorname{Inv}(\operatorname{cl}(J), f)$ which contains $0, K_{0}$, intersects the boundary of $J$.

The Carathéodory's prime ends compactification of $U_{0} \backslash K_{0}$ is a disc with a hole that we can assume to be $D^{1}=\left\{(x, y): 1 / 4 \leq x^{2}+y^{2} \leq 1\right\} \subset D$, with $D$ the Carathéodory's prime ends compactification of $S^{2} \backslash K_{0}$. The homeomorphism $f: U_{0} \backslash K_{0} \rightarrow f\left(U_{0}\right) \backslash K_{0}$ can be extended to a homeomorphism $\widehat{f}: D^{1} \rightarrow \widehat{f}\left(D^{1}\right)$. Let us observe that the set $D^{1} \backslash\left(U_{0} \backslash K_{0}\right)=\partial(D)=C_{0}$, the circle of prime ends, is invariant for $\widehat{f}$. We can suppose, after identification if necessary, that $\operatorname{Per}\left(\left.\widehat{f}\right|_{C_{0}}\right)$ is a set of two fixed points $\left\{p_{1}, p_{2}\right\}$.

We are going to see that there exists a fixed point of $\widehat{f}$ in $C_{0}$ which is asymptotically stable for $\widehat{f}$ in $D$. Let us consider an extension of $f$ to a homeomorphism of $S^{2}, f: S^{2} \rightarrow S^{2}$. Since $i_{\mathbb{R}^{2}}(f, 0)=1$, then $i_{S^{2}}\left(f, U^{c}\right)=-1$. On the other hand,

$$
1=i_{D}(\widehat{f}, D)=i_{S^{2}}\left(f, U^{c}\right)+i_{D}\left(\widehat{f}, p_{1}\right)+i_{D}\left(\widehat{f}, p_{2}\right)
$$

and $i_{D}\left(\widehat{f}, p_{i}\right) \in\{0,1\}$ (if $p_{i}$ is a repeller, $i_{D}\left(\widehat{f}, p_{i}\right)=0$, if $p_{i}$ is asymptotically stable, $i_{D}\left(\widehat{f}, p_{i}\right)=1$ and, if $p_{i}$ is a NAR point, $i_{D}\left(\widehat{f}, p_{i}\right)=1-\delta_{i}$ with $\left.\delta_{i} \in\{0,1\}\right)$. We deduce that $i_{D}\left(\widehat{f}, p_{1}\right)=i_{D}\left(\widehat{f}, p_{2}\right)=1$. A consequence is that $p_{i}$ can not be a repeller and, if $p_{i}$ is a NAR point, $\delta_{i}=0$.

If $p_{1}$ and $p_{2}$ are not asymptotically stable, then $i_{D}\left(\widehat{f}^{2}, p_{i}\right) \leq-1$ for $i=1,2$ $\left(i_{D}\left(\widehat{f}^{2}, p_{i}\right)=1-\delta_{i}-2 q_{i}\right.$ with $\delta_{i}=0$ and $\left.q_{i}>0\right)$. On the other hand, since $i_{D}\left(\widehat{f}^{2}, D\right)=1=i_{D}\left(\widehat{f}^{2}, U^{c}\right)$,

$$
i_{D}\left(\widehat{f}^{2}, p_{1}\right)+i_{D}\left(\widehat{f}^{2}, p_{2}\right)=0,
$$

but this is a contradiction. We conclude that $p_{i}$ is asymptotically stable for some $i \in\{1,2\}$.

Let us suppose, without loss of generality, that $p_{2}$ is asymptotically stable for $\widehat{f}$ with $\left(N_{2}, \emptyset\right)$ a strong filtration pair adapted to $D$ for $p_{2}$.

Let $D^{\epsilon}=\left\{(x, y): 1 / 4 \leq x^{2}+y^{2} \leq 1 / 4+\epsilon\right\} \subset D^{1}$.

Let us consider the arc $l_{2}$ contained in the external boundary of $D^{\epsilon}\left(x^{2}+y^{2}=\right.$ $1 / 4+\epsilon)$ such that $l_{2} \cap N_{2}$ are the end points of $l_{2}$. We call $n_{2} \subset \partial_{D}\left(N_{2}\right)$ the arc joining the end points of $l_{2}$ contained in $N_{2}$. The loops $L=l_{2} \cup n_{2}$ and $C_{0}$ enclose a closed disc with a hole, $V \subset D^{1}$. We select $\epsilon$ small enough such that $\widehat{f}^{2}$ and $\widehat{f}^{4}$ are defined on $V$ and $\widehat{f}^{2}(V) \cup \widehat{f}^{4}(V) \subset \operatorname{int}\left(D^{1}\right)$.

Our aim is to extend this map $\widehat{f}^{2}$, defined in the set $V$, to $D=\{(x, y)$ : $\left.1 / 4 \leq x^{2}+y^{2}<\infty\right\}$, in such a way that $\infty$ be a repeller point. Later, with an adequate perturbation of the extension, we will obtain the map $\varphi$. 


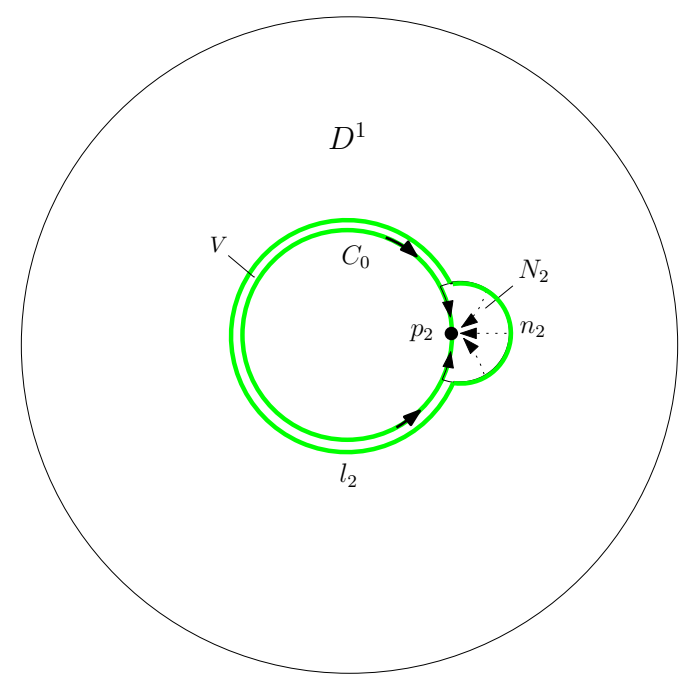

Figure 8

STEP 1. Extension of $\widehat{f}^{2}$.

The local dynamics of $\widehat{f}^{2}$ on $N_{2}$ is topologically conjugated with the dynamics of $h(x, y)=(x, y) / 2$ on the semi-disc

$$
\left\{(x, y): x^{2}+y^{2} \leq 1, y \geq 0\right\} .
$$

We suppose, without loss of generality, that $p_{2}$ has the polar coordinates $(\rho, \theta)=$ $(1 / 4,0)$ and the set $N_{2}$ is such that $d\left(p_{2}, x\right)=\delta$, with $1>\delta>\epsilon$ for every $x \in \partial_{D}\left(N_{2}\right)$.

There exists a closed arc, $I \subset N_{2}$, with end points $p_{2}$ and $q \in \partial\left(N_{2}\right) \backslash C_{0}$, positively invariant for $\widehat{f}$. On the other hand, we can select two closed arcs $I_{a}, I_{b} \subset N_{2}$, with start point $p_{2}$ and end points $a, b \in \partial\left(N_{2}\right) \backslash C_{0}$ respectively, with $a, b$ close enough to the point $q$ and such that $\widehat{f}^{2}\left(I_{a}\right) \varsubsetneqq I_{a}$ and $\widehat{f}^{2}\left(I_{b}\right) \varsubsetneqq I_{b}$. Let us assume, without loss of generality, that

$$
I=\left\{(\rho, \theta): \frac{1}{4} \geq \rho \geq \frac{1}{4}+\delta, \theta=0\right\}
$$

and the polar coordinates of $q, a$ and $b$ are, respectively, $q=(1 / 4+\delta, 0), a=$ $\left(\rho_{0},-\theta_{0}\right) \approx q, b=\left(\rho_{0}, \theta_{0}\right) \approx q$ with $\rho_{0}, \theta_{0}>0$.

Let $m=\overline{a b}$ be the arc on $\partial\left(N_{2}\right) \backslash \partial(D)$ with end points $a$ and $b$, and let $n=\widehat{f}^{2}(m)$ be the arc with end points $a_{0}=\widehat{f}^{2}(a)$ and $b_{0}=\widehat{f}^{2}(b)$. We call $I_{a a_{0}}$ and $I_{b b_{0}}$ the sub-arcs of $I_{a}$ and $I_{b}$ with end points $\left\{a, a_{0}\right\}$ and $\left\{b, b_{0}\right\}$ respectively. We can construct a closed disc, $D_{0}$, with boundary the union of the four arcs $m \cup n \cup I_{a a_{0}} \cup I_{b b_{0}}$.

In the same way we define the disc $D_{1}$ with boundary the union of four arcs $m \cup I_{a a_{1}} \cup I_{b b_{1}} \cup l$. The arc $I_{a a_{1}}$ is the segment joining $a$ with the point of polar 
coordinates $a_{1}=\left(1,-\theta_{0}\right)$. In the same way, $I_{b b_{1}}$ joins $b$ with $b_{1}=\left(1, \theta_{0}\right)$. We have $D_{0} \cap D_{1}=m$. Finally, $l$ is the arc of polar coordinates

$$
l=\left\{(\rho, \theta): \rho=1, \theta \in\left[-\theta_{0}, \theta_{0}\right]\right\}
$$

In the construction of $V$, we can suppose that $\epsilon>0$ is small enough in such a way that $\widehat{f}^{2}(V) \cap D_{0}=n$ and $\widehat{f}^{2}(V) \cap D_{1}=\emptyset$.

Let $D_{2}$ be the disc $D_{2}=\left\{(\rho, \theta): \rho \in[1,2], \theta \in\left[-\theta_{0}, \theta_{0}\right]\right\}$.

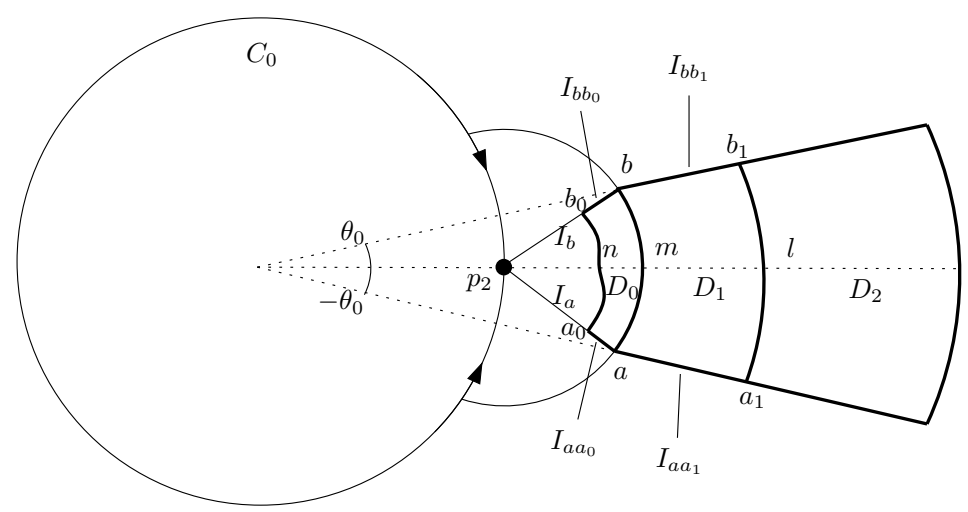

FIGURE 9

We define two orientation preserving homeomorphisms $g_{2}: D_{2} \rightarrow D_{1}$ and $g_{1}: D_{1} \rightarrow D_{0}$ in such a way that:

- $g_{2}\left(\{2\} \times\left[-\theta_{0}, \theta_{0}\right]\right)=l, g_{2}(l)=m, g_{2}\left([1,2] \times\left\{-\theta_{0}\right\}\right)=I_{a a_{1}}$ and $g_{2}([1,2] \times$ $\left.\left\{\theta_{0}\right\}\right)=I_{b b_{1}}$.

- $g_{1}(l)=m, g_{1}(m)=n, g_{1}\left(I_{a a_{1}}\right)=I_{a a_{0}}$ and $g_{1}\left(I_{b b_{1}}\right)=I_{b b_{0}}$.

Let $C_{i}=\{(\rho, \theta): \rho \in[i-1, i]\}$ for $i \geq 3$. We define the orientation preserving map

$$
g: V \cup D_{1} \cup D_{2} \cup \bigcup_{i=3}^{\infty} C_{i} \rightarrow D
$$

in the following way:

$$
g(\rho, \theta)= \begin{cases}(\rho-1, \theta) & \text { if }(\rho, \theta) \in C_{i}, i \geq 3, \\ g_{2}(\rho, \theta) & \text { if }(\rho, \theta) \in D_{2}, \\ g_{1}(\rho, \theta) & \text { if }(\rho, \theta) \in D_{1}, \\ \hat{f}^{2}(\rho, \theta) & \text { if }(\rho, \theta) \in V .\end{cases}
$$

The loop

$$
C=\partial_{D}(V) \backslash m \cup\left(\left[\rho_{0}, 2\right] \times\left\{-\theta_{0}\right\}\right) \cup\left(\left[\rho_{0}, 2\right] \times\left\{\theta_{0}\right\}\right) \cup\left(\{2\} \times\left[\theta_{0}, 2 \pi-\theta_{0}\right]\right)
$$


is the boundary of the disc $F$ in which $g$ must be extended. We extend the orientation preserving map $g$ to a homeomorphism $g: D \rightarrow D$ with $\infty$ a repeller fixed point. The extended map is selected in such a way that

$$
V \cup \widehat{f}^{2}(V) \subset \operatorname{int}_{D}\left(D_{\gamma}\right) \text { and } \operatorname{Fix}\left(\left.g\right|_{D_{\gamma} \backslash \partial(D)}\right)=\emptyset
$$

where $D_{\gamma}$ is homeomorphic to a small enough disc with a hole. The boundary of $D_{\gamma}$ is $\gamma \cup m \cup C_{0}$ with $\gamma$ a closed arc with end points $a$ and $b$, such that:

1. $\gamma \subset \operatorname{int}\left(D^{1}\right) \cap F$.

2. $\gamma \cap C=\{a, b\}$.

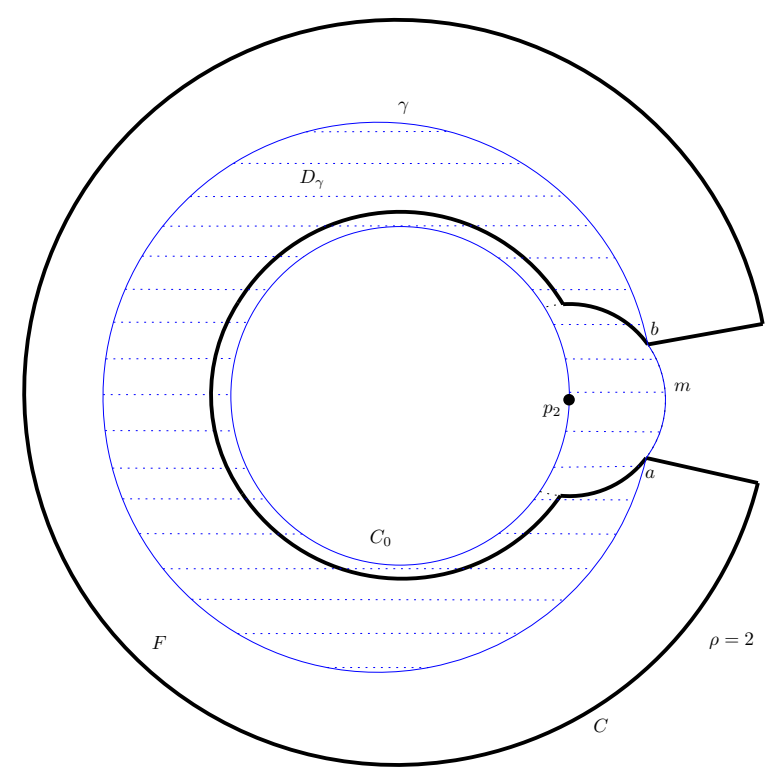

FiguRe 10

The two conditions above, $V \cup \widehat{f}^{2}(V) \subset \operatorname{int}_{D}\left(D_{\gamma}\right)$ and $\operatorname{Fix}\left(\left.g\right|_{D_{\gamma} \backslash \partial(D)}\right)=\emptyset$, are obtained in an easy way: If the extended map $g$ only satisfies the condition $V \cup \widehat{f}^{2}(V) \subset \operatorname{int}_{D}\left(D_{\gamma}\right)$, with an adequate smaller $\varepsilon>0$ in the definition of $V$, we obtain a new set, which we also call $V$, where the map $g$ satisfies the two conditions.

If we consider the orientation preserving homeomorphism $\varphi_{0}: S^{2} \rightarrow S^{2}$, induced by $g$, we have that

- $\left.\varphi_{0}\right|_{V_{0}}=\left.f^{2}\right|_{V_{0}}$ for $V_{0} \subset \mathbb{R}^{2}$ the closed disc induced by $V$. Consequently, $i_{\mathbb{R}^{2}}\left(\varphi_{0}, V_{0}\right)=1$.

- $\varphi_{0}=g$ in $S^{2} \backslash V_{0}$.

- The fixed point $\infty$ is a repeller for $\varphi_{0}$. Then $i_{S^{2}}\left(\varphi_{0}, \infty\right)=1$.

SteP 2. Perturbation of the extension $\varphi_{0}$ and construction of $\varphi$. 
First of all, let us construct a family of sets that we need in this step. Let $U^{-}$and $U^{+}$be two small enough open tubular neighbourhoods of the segments $\left[\rho_{0}, 1\right] \times\left\{-\theta_{0}\right\}$ and $\left[\rho_{0}, 1\right] \times\left\{\theta_{0}\right\}$ respectively in such a way that $\varphi_{0}(x) \neq x$ for every $x \in U^{-} \cup U^{+}$. Let $U^{\theta_{0}}$ be a small enough open tubular neighbourhood of the set $\{1\} \times\left[\theta_{0}, 2 \pi-\theta_{0}\right]$, and let $V^{\theta_{0}}$ be a small enough open tubular neighbourhood of the set $[1 / 4,1] \times\left[\theta_{0}, 2 \pi-\theta_{0}\right]$. Let us call $W=\operatorname{int}\left(D_{\gamma}\right) \cup U^{-} \cup U^{+}$.

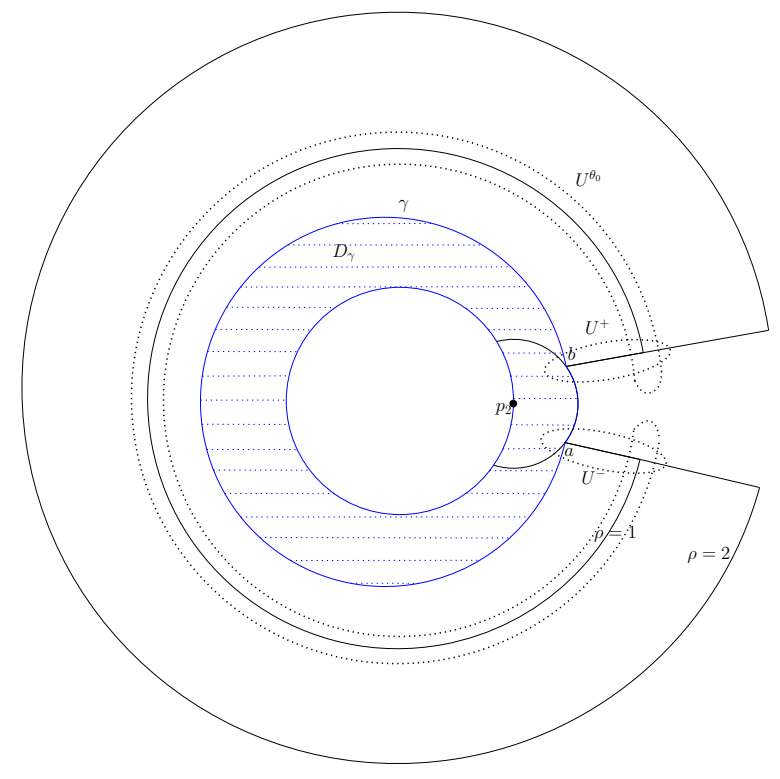

FIGURE 11

The sets $U^{+}, U^{-}, U^{\theta_{0}}, V^{\theta_{0}}$ and $W$ above have the following properties:

Since $\varphi_{0}(F) \subset[1 / 4,1] \times[0,2 \pi]$ and $F \subset[1 / 4,2] \times\left[\theta_{0}, 2 \pi-\theta_{0}\right]$ (in polar coordinates), then

$$
F \cap \varphi_{0}(F) \subset V^{\theta_{0}} .
$$

On the other hand, let $x \in C \cap V^{\theta_{0}}$. If $x \notin U^{+} \cup U^{-}$, then $x \in \operatorname{int}\left(D_{\gamma}\right)$. Consequently,

$$
C \cap V^{\theta_{0}} \subset W
$$

Let us suppose that $x \in C$. If $x \in \partial_{D}(V) \backslash m$, then $\varphi_{0}(x) \in \operatorname{int}\left(D_{\gamma}\right)$. If $x=\left(\rho, \pm \theta_{0}\right)$ with $\rho \in\left[\rho_{0}, 2\right]$, then $\varphi_{0}(x) \in U^{+} \cup U^{-} \cup \operatorname{int}\left(D_{\gamma}\right)$. If $x=(2, \theta)$, then $\varphi_{0}(x) \in U^{\theta_{0}}$. Consequently,

$$
\varphi_{0}(C) \subset U^{\theta_{0}} \cup W
$$

Finally,

$$
\left(U^{\theta_{0}} \backslash W\right) \cap \varphi_{0}(C) \text { is an arc } \gamma_{1} \subset F \text {. }
$$


A consequence of the last observations of the Step 1 is that $i_{\mathbb{R}^{2}}\left(\varphi_{0}, F\right)=0$. Our aim is to apply a theorem of Schmitt (see [34]) to $\varphi_{0}$ in order to get rid of the fixed points of $\left.\varphi_{0}\right|_{F}$. Schmitt's theorem says that if $F \cap \varphi_{0}(F)$ is a finite union of disjoint discs, $F \cap \varphi_{0}(F)=\bigcup_{i=1, \ldots, k} F_{i}$, and $i_{\mathbb{R}^{2}}\left(\varphi_{0}, F_{i}\right)=0$ for $i=$ $1, \ldots, k$, it is possible to extend $\left.\varphi_{0}\right|_{C}$ to the closed $\operatorname{disc} F$ in such a way that $\operatorname{Fix}\left(\left.\varphi_{0}\right|_{F}\right)=\emptyset$. But $F \cap \varphi_{0}(F)$ could not be a finite union of disjoint discs. To avoid this problem, we take a slight modification of the map $\varphi_{0}$ in a closed disc $C_{\varepsilon^{\prime}} \subset \operatorname{int}(D)$ which is a small enough tubular neighbourhood of $C,\left(\varepsilon^{\prime}<\varepsilon\right)$. Let $\varphi_{0}^{*}$ this homeomorphism in such a way that the properties (3.1)-(3.4) above remains true and:

- $\left.\varphi_{0}\right|_{S^{2} \backslash C_{\varepsilon^{\prime}}}=\left.\varphi_{0}^{*}\right|_{S^{2} \backslash C_{\varepsilon^{\prime}}}$ with $\operatorname{Fix}\left(\left.\varphi_{0}^{*}\right|_{C_{\varepsilon^{\prime}}}\right)=\emptyset$.

- $C$ is transversal to $\varphi_{0}^{*}(C)$ with $F \cap \varphi_{0}^{*}(F)$ a finite union of disjoint discs, $F \cap \varphi_{0}^{*}(F)=\bigcup_{i=1, \ldots, k} F_{i}$.

We shall prove that $i_{\mathbb{R}^{2}}\left(\varphi_{0}^{*}, F_{i}\right)=0$ for $i=1, \ldots, k$.

Let $F_{i}$ be fixed. One has that $C_{i}=\partial\left(F_{i}\right)$ is the alternate union of arcs obtained from $C$ and $\varphi_{0}^{*}(C)$, that is, $C_{i}=\bigcup_{j=1}^{k_{i}}\left(G_{j} \cup H_{j}\right)$ with $G_{j} \subset C$ and $H_{j} \subset \varphi_{0}^{*}(C)$ homeomorphic to closed intervals such that $G_{j} \cap H_{j}$ is a point and $H_{j} \cap G_{j+1}$ is a point.

If $F_{i} \subset W$, then for all $x \in F_{i} \cap\left(U^{+} \cup U^{-}\right)$we have $\varphi_{0}^{*}(x) \neq x$. On the other hand, if $x \in F_{i} \cap\left(\operatorname{int}\left(D_{\gamma}\right) \backslash C_{\varepsilon^{\prime}}\right)$, then $\varphi_{0}^{*}(x)=\varphi_{0}(x) \neq x$ and if $x \in F_{i} \cap \operatorname{int}\left(D_{\gamma}\right) \cap C_{\varepsilon^{\prime}}$, then $\varphi_{0}^{*}(x) \neq x$. Consequently, $i_{\mathbb{R}^{2}}\left(\varphi_{0}^{*}, F_{i}\right)=0$.

If $F_{i} \not \subset W$, then $C_{i} \not \subset W$ and there exists $x \in C_{i} \backslash W$. We have, by (3.1), that $x \in V^{\theta_{0}}$ and, since $x \in G_{j}$ or $x \in H_{j}$ it is only possible that $x \in H_{j}$ because, if $x \in G_{j} \subset C$, then, by (3.2), $x \in W$, which is a contradiction. Because of (3.3),

$$
x \in H_{j} \subset \varphi_{0}^{*}(C) \subset U^{\theta_{0}} \cup W
$$

Since $x \notin W$, we deduce that $x \in U^{\theta_{0}} \backslash W$. By $(3.4),\left(U^{\theta_{0}} \backslash W\right) \cap \varphi_{0}^{*}(C)$ is an arc $\gamma_{1}$, and $x \in \gamma_{1}$. Moreover, $\gamma_{1} \subset C_{i} \subset F_{i}$ which proves that $F_{i} \not \subset W$ exists and is unique. Let us call it $F_{0}$. By the last three observations of the Step 1 and, by the construction of $\varphi_{0}^{*}$, we have that $i_{\mathbb{R}^{2}}\left(\varphi_{0}^{*}, F\right)=0$. On the other hand, $i_{\mathbb{R}^{2}}\left(\varphi_{0}^{*}, F_{i}\right)=0$ for every $F_{i} \subset W$. Consequently, $i_{\mathbb{R}^{2}}\left(\varphi_{0}^{*}, F_{0}\right)=0$ and we are in the conditions of the theorem of Schmitt which permit us to modify $\varphi_{0}^{*}$ in $F$ in order to obtain the homeomorphism $\varphi: \mathbb{R}^{2} \rightarrow \mathbb{R}^{2}$ we are looking for. Let us remember that $\varphi$ is dissipative, $\operatorname{Fix}(\varphi)=\{0\}$, and $\left.\varphi\right|_{W_{0}}=\left.f^{2}\right|_{W_{0}}$ with $W_{0}$ a neighbourhood of 0 . It only remains to prove that $\{0\}$ is a global attractor for $\varphi$.

STEP 3. $\{0\}$ is a global attractor for $\varphi$.

Let us consider $x \in \mathbb{R}^{2} \backslash\{0\}$. The theorem is proved if we see that $\omega_{\varphi}(x)=0$. 
Let $J$ be a small enough Jordan domain, $0 \in J$, with $x \notin \operatorname{cl}(J)$ and let $\widehat{\varphi}: D \rightarrow D$ be the map induced by $\varphi$. The $\operatorname{disc} D$ is the Carathéodory's compactification of $S^{2} \backslash K_{0}$, with $K_{0}$ the connected component of $\operatorname{Inv}(\operatorname{cl}(J), \varphi)$ which contains $\{0\}$. We consider a closed arc, $\Gamma \subset D$, invariant for $\widehat{\varphi}$, with extreme points $p_{2}$ and $\infty$, such that $\Gamma \cap \partial(D)=\left\{p_{2}\right\}$ and such that $\varphi^{k}(y) \rightarrow p_{2}$ if $k \rightarrow \infty$ for all $y \in \Gamma \backslash\{\infty\}$.

Let us take the universal covering $E_{0}=\{(x, y): y \geq 0\}$ of $B_{0}=D \backslash\{\infty\}$, with covering map $p: E_{0} \rightarrow B_{0}$ and let us consider the homeomorphism $\varphi_{E_{0}}: E_{0} \rightarrow E_{0}$ induced by $\varphi_{B_{0}}: B_{0} \rightarrow B_{0}$ (with $\varphi_{B_{0}}=\left.\widehat{\varphi}\right|_{B_{0}}$ ) such that $p \circ \varphi_{E_{0}}=\varphi_{B_{0}} \circ p$ and such that sends each point in $p^{-1}\left(p_{2}\right)$ to itself.

We will also work with the universal covering $E=\{(x, y): y>0\}$ of $B=D \backslash$ $(\partial(D) \cup\{\infty\})$, with covering map $p: E \rightarrow B$ as above, and the homeomorphisms $\varphi_{E}: E \rightarrow E$ and $\varphi_{B}: B \rightarrow B$.

We consider the sets

$p^{-1}\left(p_{2}\right)=\bigcup_{i \in \mathbb{Z}} r_{i} \subset E_{0}, \quad p^{-1}(\Gamma)=\bigcup_{i \in \mathbb{Z}} \Gamma_{r_{i}} \subset E_{0}, \quad p^{-1}(D \backslash \Gamma)=\bigcup_{i \in \mathbb{Z}} \Delta_{r_{i}} \subset E_{0}$

with $\Gamma_{r_{i}}$ and $\Delta_{r_{i}}$ the connected components of $p^{-1}(\Gamma)$ and $p^{-1}(D \backslash \Gamma)$ respectively, and $r_{i}-r_{i-1}=2 \pi$. The sets $\Gamma_{r_{i}}$ and $\Delta_{r_{i}}$ are unbounded and invariant for $\varphi_{E_{0}}$.

The omega-limit set of $x$ for $\varphi_{B}, \omega_{\varphi_{B}}(x)$, is a closed subset of $B$, invariant for $\varphi_{B}$. We claim that $\omega_{\varphi_{B}}(x)=\emptyset$.

Indeed, let us suppose that $x \in D \backslash \Gamma$ (if $x \in \Gamma$, the proof is trivial). We can select a closed arc $C_{x}$ joining $x$ with $p_{2} \in \partial(D)$ and such that $C_{x} \backslash\left\{p_{2}\right\} \subset D \backslash \Gamma$. Let $p^{-1}\left(C_{x}\right)=\bigcup_{i \in \mathbb{Z}} C_{r_{i}}$, with $C_{r_{i}}$ the connected components. Let us observe that $C_{r_{i}} \backslash\left\{r_{i}\right\} \subset \Delta_{r_{i}}$. We take $x_{0} \in p^{-1}(x), r_{0} \in p^{-1}\left(p_{2}\right)$ and the component $C_{r_{0}}$ of $p^{-1}\left(C_{x}\right)$ joining the two points. As $C_{x}$ is bounded, there exists $l_{x}>0$ such that the projection on the coordinate $y, \Pi_{y}(z)$ is less or equal to $l_{x}$ for all $z \in p^{-1}\left(C_{x}\right)$. On the other hand, as $\infty$ is a repeller point for $\varphi$, the iterations by $\varphi_{E_{0}}$ of $x_{0}$ are bounded by a number $m_{x}>0$ which depends on $x$, that is, $\Pi_{y}\left(\varphi_{E_{0}}{ }^{k}\left(x_{0}\right)\right) \leq m_{x}$ for every $k \in \mathbb{N}$. Moreover, we can select $m_{x}$ such that $\Pi_{y}\left(\varphi_{E_{0}}{ }^{k}(z)\right) \leq m_{x}$ for every $z \in E_{0}$ such that $\Pi_{y}(z) \leq l_{x}$.

Since $\left\{y=m_{x}\right\} \cap \Gamma_{r_{0}} \neq \emptyset$ and $\left\{y=m_{x}\right\} \cap \Gamma_{r_{1}} \neq \emptyset$, it is easy to select two points $y_{r_{0}} \in \Gamma_{r_{0}} \cap\left\{y=m_{x}\right\}$ and $y_{r_{1}} \in \Gamma_{r_{1}} \cap\left\{y=m_{x}\right\}$ and two closed arcs $\gamma_{r_{0}} \subset \Gamma_{r_{0}}, \gamma_{r_{1}} \subset \Gamma_{r_{1}}$, homeomorphic to $[0,1]$, with extreme points $\left\{y_{r_{0}}, r_{0}\right\}$ and $\left\{y_{r_{1}}, r_{1}\right\}$ respectively such that

$$
\left(\gamma_{r_{0}} \cup \gamma_{r_{1}}\right) \cap\left\{y=m_{x}\right\}=\left\{y_{r_{0}}, y_{r_{1}}\right\} .
$$

Since the sets $\varphi_{E_{0}}^{k}\left(C_{r_{0}} \backslash\left\{r_{0}\right\}\right)$ stay in $\Delta_{r_{0}}$ for all $k \in \mathbb{Z}$ and $\Pi_{y}\left(\varphi_{E_{0}}^{k}\left(C_{r_{0}}\right)\right) \leq$ $m_{x}$ for all $k \in \mathbb{N}$, we obtain that $\varphi_{E_{0}}^{k}\left(C_{r_{0}}\right) \subset R$ for all $k \in \mathbb{N}$, where $R \subset E_{0}$ is homeomorphic to a closed disc bounded by $\left[r_{0}, r_{1}\right] \cup\left[y_{r_{0}}, y_{r_{1}}\right] \cup \gamma_{r_{0}} \cup \gamma_{r_{1}}$. 

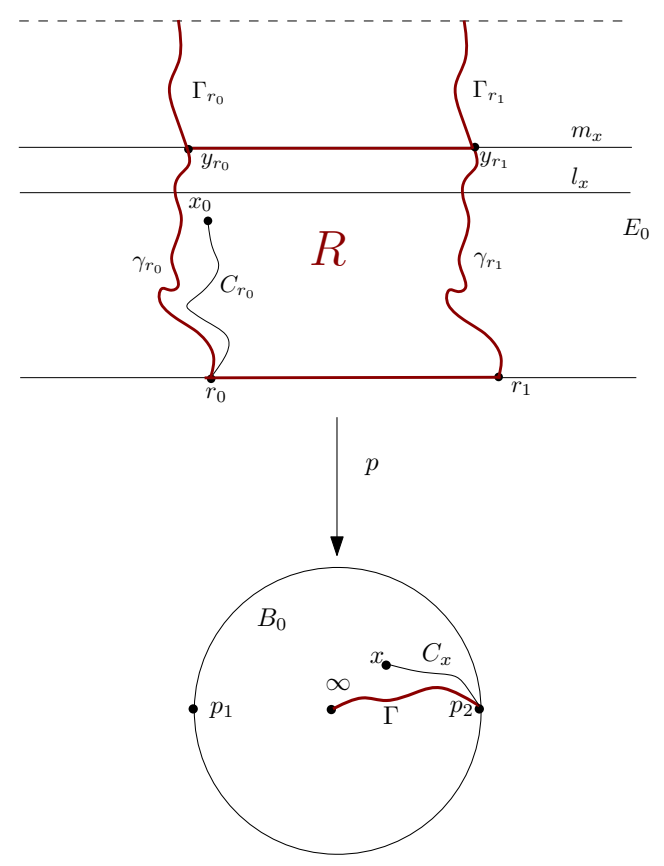

Figure 12

Let $r_{m}<\Pi_{x}(z)$ for all $z \in \gamma_{r_{0}}$ and $r_{n}>\Pi_{x}(z)$ for all $z \in \gamma_{r_{1}}$. The rectangle $\left[r_{m}, r_{n}\right] \times\left(0, m_{x}\right]$ contains the positive semi-orbit $\left\{\varphi_{E}^{k}\left(x_{0}\right)\right\}_{k \in \mathbb{N}}$. If the omegalimit set $\omega_{\varphi_{E}}\left(x_{0}\right) \neq \emptyset$, by the Brouwer's Lemma on translation arcs, there exists a fixed point $x_{1}$ for $\varphi_{E}$ in $E$ and then $p\left(x_{1}\right) \in B$ is a fixed point for $\varphi_{B}$ which is a contradiction.

Since the last argument is valid for every small enough Jordan domain $J$ and $\omega_{\varphi}(x) \subset \mathbb{R}^{2}$ is non-empty because $\infty$ is a repeller, we obtain that $\{0\}=\omega_{\varphi}(x)$ for every $x \in \mathbb{R}^{2}$ which finishes the proof of the theorem.

REMARK 3.1. The converse of the above Theorem 1.1 says that if there exists the homeomorphism $\varphi$, then $i_{\mathbb{R}^{2}}\left(f^{2}, 0\right)=1$ and $i_{\mathbb{R}^{2}}(f, 0) \in\{-1,1\}$.

Indeed, since $i_{S^{2}}(\varphi, 0)+i_{S^{2}}(\varphi, \infty)=2$, then $i_{\mathbb{R}^{2}}\left(f^{2}, 0\right)=1$. If 0 is stable, we have $i_{\mathbb{R}^{2}}(f, 0)=1$. On the other hand, if 0 is not stable, $i_{\mathbb{R}^{2}}\left(f^{2}, 0\right)=1=$ $1-u_{0}+r_{0}$. The numbers $u_{0}=r_{0}$ are the number of exit components and repellers for $\widehat{f}^{2}$ at the periodic points of $\partial(D)$ respectively (see Theorem 2.15). Moreover, $i_{\mathbb{R}^{2}}(f, 0)=1-u_{0}^{\prime}-r_{0}^{\prime}$ with $0 \leq u_{0}^{\prime}+r_{0}^{\prime} \leq 2$. The number $u_{0}^{\prime}$ is the number of exit components for $\widehat{f}$ at the fixed points $\left\{p_{1}, p_{2}\right\}$ which give us fixed points, after identification to points, for the quotient map $\bar{f}$. The number $r_{0}^{\prime}$ is the number of repeller fixed points of $\widehat{f}$ in $\partial(D)$. Let us see that $u_{0}^{\prime}+r_{0}^{\prime}=1$ is not possible. In fact, if it is true, then there exists a fixed point of $\partial(D)$, for 
instance, $p_{1}$, which is a repeller or NAR with an exit component for $\widehat{f}$ which is invariant, after identification to points, for the quotient map $\overline{\widehat{f}}$. The other fixed point, $p_{2}$, can not be a repeller and has no exit components as above. But in this situation it is impossible that $u_{0}=r_{0}$ because one of them is odd and the other one is even.

Let us see an example of the remark above with $i_{\mathbb{R}^{2}}(f, 0)=-1$.

ExAmple 3.2. Let us consider the dynamical system $\varphi: S^{2} \rightarrow S^{2}$ of Figure 13 .

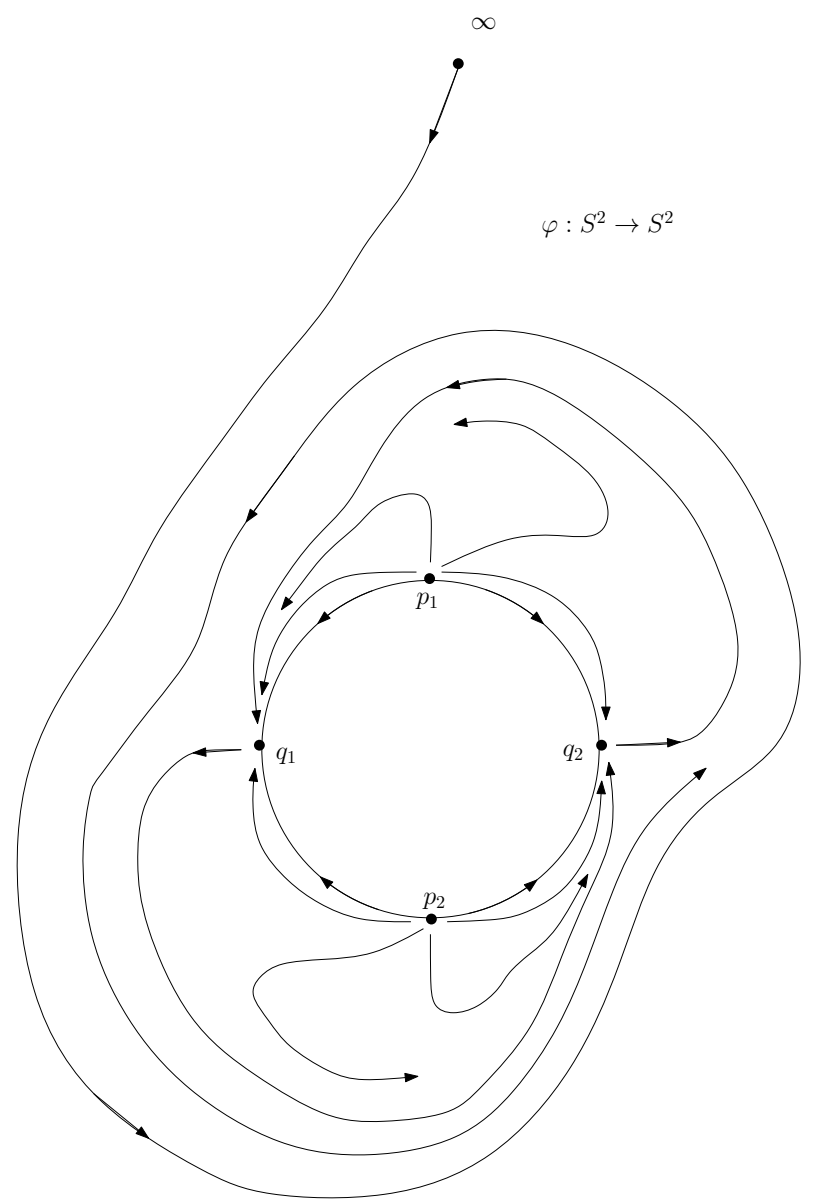

FIGURE 13. $\widehat{f}\left(p_{1}\right)=p_{1}, \widehat{f}\left(p_{2}\right)=p_{2}, \widehat{f}\left(q_{1}\right)=q_{2}, \widehat{f}\left(q_{2}\right)=q_{1}, i(f, 0)=$ $1-u_{0}^{\prime}-r_{0}^{\prime}=-1, i\left(f^{2}, 0\right)=1-u_{0}+r_{0}=1$.

It is clear that $i_{\mathbb{R}^{2}}(f, 0)=1-u_{0}^{\prime}-r_{0}^{\prime}=1-0-2=-1$.

Corollary 3.3. Let $f: U \subset \mathbb{R}^{2} \rightarrow f(U) \subset \mathbb{R}^{2}$ be an orientation reversing homeomorphism with $\operatorname{Fix}(f)=\operatorname{Fix}\left(f^{2}\right)=\{0\}$. Then, if $\{0\}$ is stable, it is asymptotically stable. 
Proof. In fact, if $\{0\}$ is stable, $i_{\mathbb{R}^{2}}(f, 0)=i_{\mathbb{R}^{2}}\left(f^{2}, 0\right)=1$ and, by the last theorem, $\{0\}$ is a global attractor for an extension $\varphi: S^{2} \rightarrow S^{2}$ of $f^{2}$. Then, $\{0\}$ is asymptotically stable for $\varphi$ and, consequently, it is asymptotically stable for $f$.

\section{An application to periodic differential equations with symmetries}

Now we show how orientation-reversing maps appear naturally in the context of certain periodic differential equations with symmetries.

Let $S$ denote the symmetry $S\left(\left(x_{1}, x_{2}\right)\right)=\left(x_{1},-x_{2}\right)$ and consider the differential system

$$
\dot{x}=F(t, x), \quad x \in \mathbb{R}^{2}
$$

where $F: \mathbb{R} \times \mathbb{R}^{2} \rightarrow \mathbb{R}^{2}$ satisfies

$$
F(t+\pi, S(x))=S(F(t, x)), \quad \text { for each }(t, x) \in \mathbb{R} \times \mathbb{R}^{2} .
$$

We shall assume that the vector field $F$ is continuous and that there is global existence and uniqueness for the initial value problem. The solution satisfying the initial condition $x\left(t_{0}\right)=x_{0}$ will be denoted by $x\left(t ; t_{0}, x_{0}\right)$ and it is well defined for all $t \in \mathbb{R}$.

Now we consider the maps $P_{1}\left(x_{0}\right)=x\left(\pi ; 0, x_{0}\right)$ and $P_{2}\left(x_{0}\right)=x\left(2 \pi ; \pi, x_{0}\right)$. One has that $S \circ P_{1}=P_{2} \circ S$. In fact, if $x(t)$ is a solution then $S(x(t+\pi))$ is also a solution. Then

$$
x\left(t ; 0, S\left(x_{0}\right)\right)=S\left(x\left(t+\pi ; \pi, x_{0}\right)\right) .
$$

This implies that $S \circ P_{2}=P_{1} \circ S$.

From the symmetry of the vector field we have that the system is $2 \pi$-periodic with respect to time and the Poincaré map $P\left(x_{0}\right)=x\left(2 \pi ; 0, x_{0}\right)$ satisfies

$$
P=P_{2} \circ P_{1}=P_{2} \circ S \circ S \circ P_{1}=\left(S \circ P_{1}\right)^{2} .
$$

It is well known that the maps $P_{1}, P_{2}$ and $P$ preserve the orientation. Then, $P$ can be decompose as $P=r \circ r$ where $r=S \circ P_{1}$ reverses the orientation.

COROllary 4.1. Consider the system in the above conditions and assume in addition that $F$ is $C^{1}, F(t, 0)=0$ for all $t$ and the trivial solution $x=0$ is isolated as $2 \pi$-periodic orbit and it is Lyapunov stable. Then:

(a) The trivial solution $x=0$ is asymptotically stable.

(b) If the system is dissipative, the trivial solution $x=0$ is globally asymptotically stable if and only if there are no more $2 \pi$-periodic solutions.

Proof. Since $i_{\mathbb{R}^{2}}(r, 0)=i_{\mathbb{R}^{2}}\left(r^{2}, 0\right)=1$ (see [10] and [29]) the first assertion is an immediate consequence of Corollary 3.3 applied to $r$. 
In order to prove (b), let us assume that the trivial solution $x=0$ is locally asymptotically stable. That means that 0 is a local asymptotically stable attractor for the Poincaré map $P=r^{2}$. Let $U$ be its basin of attraction. We have that $U \neq \mathbb{R}^{2}$ is a simply connected open set such that $\partial(U) \neq \emptyset$. If $U$ is bounded, a result of Kuperberg (see [17]) implies that $\operatorname{Fix}(P) \cap \partial(U) \neq \emptyset$. Otherwise we apply Propositions 2 and 6 of [26] to get a fixed point of $P=r^{2}$ in $\mathbb{R}^{2} \backslash U$.

\section{REFERENCES}

[1] J.M. Alonso And J. CAmpos, The index and the asymptotic stability of fixed points in two dimensions: a counterexample, Nonlinear Anal. 32 (1998), 719-725.

[2] M. Barge and R.M. Gillette, A fixed point theorem for plane separating continua, Topology Appl. 43 (1992), 203-212.

[3] C. Bonatti And B. Kolev, Surface homeomorphisms with zero-dimensional singular set, Topology Appl. 90 (1998) 69-95.

[4] L.E. Brouwer, Beweis des ebenen translationssatzes, Math. Annalen 72 (1912), 37-54.

[5] R.F. Brown, The Lefschetz Fixed Point Theorem, Scott Foreman Co. Glenview Illinois, London, 1971.

[6] C. Carathéodory, Über die Begrenzung einfach zusammenhängender Gebiete, Math. Ann. 73 (1913), 323-370.

[7] M.L.Cartwright and J.E. Littlewood, Some fixed point theorems, Ann. of Math. 54, 1, (1951), 1-37.

[8] C.O. Christenson and W.L. Voxman, Aspects of Topology, BCS Associates, Moscow, Idaho, 1998.

[9] E.N. DANCER, On the indices of fixed points of mappings in cones and applications, J. Math. Anal. Appl. 91 (1983), 131-151.

[10] E.N. Dancer And R. Ortega, The index of Lyapunov stable fixed points, J. Dynam. Differential Equations 6 (1994), 631-637.

[11] A. DoLD, Fixed point index and fixed point theorem for Euclidean neighborhood retracts, Topology 4 (1965), 1-8.

[12] J. Franks and D. Richeson, Shift equivalence and the Conley index, Trans. Amer. Math. Soc. 352, 7 (2000), 3305-3322.

[13] G. Graff and P. Nowak-Przygodzki, Fixed point indices of the iterations of planar homeomorphisms, Topol. Methods Nonlinear Anal. 22 (2003), 159-166.

[14] J. Jezierski and W. Marzantowicz, Homotopy Methods in Topological Fixed and Periodic Point Theory, Springer, 2005.

[15] B. KerÉKJÁrTó, Voresungen Uber Topologie (I), Springer, Berlin, 1923.

[16] _ Sur le caractèr topologique des représentations conformes, C.R. Acad. Sci. 198 (1934), 317-320.

[17] K. KuPERBerg, Fixed points of orientation reversing homeomorphisms of the plane, Proc. Amer. Math. Soc. 112 (1991), 1, 223-229.

[18] P. Le Calvez, Une propriété dynamique des homéomorphismes du plan au voisinage d'un point fixe d'indice > 1, Topology 38, 1 (1999), 23-35.

[19] _ Dynamique des homéomorphismes du plan au voisinage d'un point fixe. Ann. Sci. Ecole Norm. Sup. (4) 36 (2003), no. 1, 139-171.

[20] P. Le Calvez and J.-C. Yoccoz, Un théoréme d'indice pour les homéomorphismes du plan au voisinage d'un point fixe, Ann. of Math. 146 (1997), 241-293. 
[21] F. LE Roux, Homéomorphismes de surfaces - Théorèmes de la fleur de Leau-Fatou et de la variété stable, Astérisque 292 (2004).

[22] J.N. MATHER, Topological proofs of some purely topological consequences of Carathéodory's theory of prime ends. Selected studies: physics-astrophysics, mathematics, history of science, North-Holland, Amsterdam-New York, 1982, pp. 225-255.

[23] J.R. Munkres, Topology, Prentice Hall, 2000

[24] R.D. Nussbaum, The fixed point index and some applications, Séminaire de Mathématiques supérieures, Les Presses de L'Université de Montréal, 1985.

[25] R. ORtegA, A criterion for asymptotic stability based on topological degree, In Proceedings of the First World Congress of Nonlinear Analysts, Tampa (1992), 383-394.

[26] R. Ortega And F.R. Ruiz Del Portal, Attractors with vanishing rotation number, J. Eur. Math. Soc. 13 (2011), 1567-1588.

[27] Ch. Pommerenke, Boundary Behavior of Conformal Maps, Grundlehren der mathematischen Wissenschaften 299, 1992, Springer-Verlag, Berlin, Heidelberg, New York.

[28] S. Pelikan and E.E. Slaminka, A bound for the fixed point index of area-preserving homeomorphisms of two-manifolds, Ergodic Theory Dynam. Systems 7 (1987), 463-479.

[29] F.R. Ruiz Del Portal, Planar isolated and stable fixed points have index=1, J. Differential Equations 199 (2004), 179-188.

[30] F.R. Ruiz del Portal and J.M. Salazar, Fixed point index of iterations of local homeomorphisms of the plane: a Conley-index approach, Topology 41 (2002), 1199-1212.

[31] _ A stable/unstable manifold theorem for local homeomorphisms of the plane, Ergodic Theory Dynam. Systems 25 (2005), 301-317.

[32] _ A Poincaré formula for the fixed point indices of the iterates of arbitrary planar homeomorphisms, Fixed Point Theory Appl. (2010), ID 323069, 1-31.

[33] C.P. Simon, A bound for the fixed point index of an area-preserving map with applications to mechanics, Inventiones Math. 26 (1974), 187-200.

[34] B. Schmitt, Sur les plongements, admettant zero ou un point fixe, du disque dans le plan, Topology 14 (1974), 357-365.

Francisco R. Ruiz del Portal

Departamento de Geometría y Topología

Facultad de CC.Matemáticas

Universidad Complutense de Madrid

Madrid 28040, SPAIN

E-mail address: R_Portal@mat.ucm.es

José Manuel SAlazar

Departamento de Física y Matemáticas

Universidad de Alcalá

Alcalá de Henares

Madrid 28871, SPAIN

E-mail address: josem.salazar@uah.es 